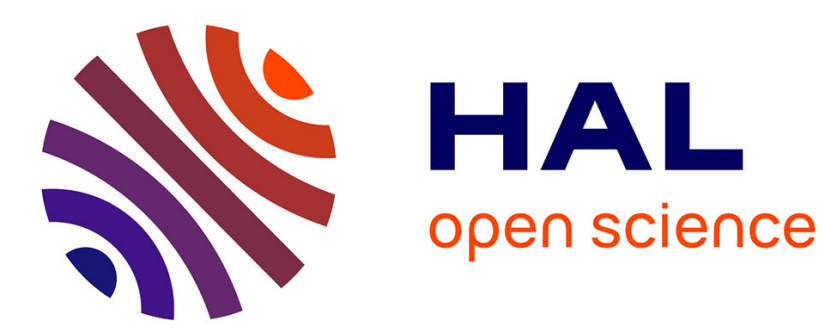

\title{
On residual properties of pure braid groups of closed surfaces
}

\author{
Valerij G. Bardakov, Paolo Bellingeri
}

\section{To cite this version:}

Valerij G. Bardakov, Paolo Bellingeri. On residual properties of pure braid groups of closed surfaces. 2007. hal-00150073

\section{HAL Id: hal-00150073 \\ https://hal.science/hal-00150073}

Preprint submitted on 29 May 2007

HAL is a multi-disciplinary open access archive for the deposit and dissemination of scientific research documents, whether they are published or not. The documents may come from teaching and research institutions in France or abroad, or from public or private research centers.
L'archive ouverte pluridisciplinaire HAL, est destinée au dépôt et à la diffusion de documents scientifiques de niveau recherche, publiés ou non, émanant des établissements d'enseignement et de recherche français ou étrangers, des laboratoires publics ou privés. 


\title{
ON RESIDUAL PROPERTIES OF PURE BRAID GROUPS OF CLOSED SURFACES
}

\author{
VALERIJ G. BARDAKOV AND PAOLO BELLINGERI
}

\begin{abstract}
We prove that pure braid groups of closed surface are almost-direct products of residually torsion free nilpotent groups and hence residually torsion free nilpotent. As a Corollary, we prove also that braid groups on 2 strands of closed surfaces are residually nilpotent.
\end{abstract}

\section{INTRODUCTION}

A group $G$ is said to be residually torsion free nilpotent if for any (non-trivial) element $x \in G$, there exists a homomorphism $\phi: G \rightarrow H$ such that $H$ is torsion free nilpotent and $\phi(x) \neq 1$.

Let $A, C$ be two groups. If $C$ acts on $A$ by automorphisms, the semi-direct product $A \rtimes C$ is said to be almost-direct if the action of $C$ on the abelianization of $A$ is trivial. An example of almost-direct product of free groups is given by Artin pure braid group $P_{n}$. Such decomposition implies that $P_{n}$ is residually torsion-free nilpotent (see Section 2).

The structure of almost-direct product turns out to be also a powerful tool also in the determination of algebras related to lower central series (see for instance CCP) and more generally in the study of finite type invariants. The decomposition of $P_{n}$ as almost-direct product of free groups was used in $[\mathrm{P}]$ in order to construct an universal finite type invariant for braids with integers coefficients.

Let $\Sigma$ be an oriented surface and let $\mathbb{F}_{n}(\Sigma)=\left\{\left(x_{1}, \ldots x_{n}\right) \in \Sigma^{n} \mid x_{i} \neq\right.$ $x_{j}$ for $\left.i \neq j\right\}$. The fundamental group of $\mathbb{F}_{n}(\Sigma)$ is called pure braid group on $n$ strands of $\Sigma$ and it is usually denoted by $P_{n}(\Sigma)$. When $\Sigma$ is the disk $D^{2}$ we obtain a group which is isomorphic to Artin pure braid group $P_{n}$.

The symmetric group $S_{n}$ acts on $\mathbb{F}_{n}(\Sigma)$ by permutation of coordinates and the fundamental group of the orbit space $\mathbb{F}_{n}(\Sigma) / S_{n}$ is called braid group on $n$ strands of $\Sigma$ and it is denoted by $B_{n}(\Sigma)$. For $n=1$

1991 Mathematics Subject Classification. Primary $20 \mathrm{~F} 36$.

Key words and phrases. Braid groups, residual properties. 
we have that $P_{1}(\Sigma)=B_{1}(\Sigma)=\pi_{1}(\Sigma)$ and $B_{n}\left(D^{2}\right)$ is isomorphic to Artin braid group $B_{n}$.

When $\Sigma$ is an oriented surface of positive genus, $P_{n}$ embeds naturally into $P_{n}(\Sigma)$. In GP González-Meneses and Paris proved that the normal closure of the classical pure braid group $P_{n}$ in $P_{n}(\Sigma)$ is an almost-direct product of (infinitely generated) free groups. Adapting the approach of Papadima, they constructed a universal finite type invariant for surface braids.

At our knowledge, it is not known if pure braid groups of surfaces (different from the disk) can be decomposed as almost-direct products of (residually) free groups (see for instance [B, GG] for more details on this subject). In BGG the first author proved that pure braid groups of the torus and of surfaces with boundary components are residually torsion-free nilpotent by showing that they may be realised as subgroups of the Torelli group of a surface of higher genus (see also the end of Section 4 ).

In this paper we complete the study of lower central series and related residual properties of (pure) braid groups of surfaces begun in BGG, proving that pure braid groups of closed surface are almostdirect products of residually torsion free nilpotent groups and hence residually torsion free nilpotent (Theorem 6). As a Corollary, we prove also that braid groups on 2 strands of closed surfaces are residually nilpotent (Corollary 10).

The fact that a group is residually torsion-free nilpotent has several consequences, notably that the group is bi-orderable [MR and residually $p$-finite $\mathrm{Gr}$. Therefore it follows from Theorem 6 that pure braid groups of closed oriented surfaces are bi-orderable and residually $p$ finite; the first result was earlier proved in G0 and the second is also a consequence of Theorem $1.2 \mathrm{in}[\mathrm{Pa}]$ (see Section [1).

Acknowledgments. The research of the first author has been supported by the University of Nantes. The first author would like to thank the members of the Department of Mathematics of the University of Nantes for their kind hospitality.

\section{Residual PROPERTIES, ALMOST-DiRECT PRODUCTS AND GROUP PRESENTATIONS FOR PURE BRAID GROUPS ON CLOSED SURFACES}

Let us begin with few definitions.

The lower central series of a group $G$ is the filtration $\Gamma_{1}(G)=G \supseteq$ $\Gamma_{2}(G) \supseteq \ldots$, where $\Gamma_{i}(G)=\left[\Gamma_{i-1}(G), G\right]$. The rational lower central series of $G$ is the filtration $D_{1}(G) \supseteq D_{2}(G) \supseteq$.. obtained setting 
ON RESIDUAL PROPERTIES OF PURE BRAID GROUPS OF CLOSED SURFACEฐ

$D_{1}(G)=G$, and for $i \geq 2$, defining $D_{i}(G)=\left\{x \in G \mid x^{n} \in \Gamma_{i}(G)\right.$ for some $n \in \mathbb{N} \backslash\{0\}\}$.

Let $\mathcal{F P}$ be the family of groups having the group-theoretic property $\mathcal{P}$. A group $G$ is said to be residually $\mathcal{P}$ if for any element $x \in G \backslash 1$, there exists a homomorphism of $G$ into some group in $\mathcal{F P}$ taking $x$ in a nontrivial element.

A group $G$ is residually nilpotent if and only if $\bigcap_{i>1} \Gamma_{i}(G)=\{1\}$. On the other hand, a group $G$ is residually torsion-free nilpotent if and only if $\bigcap_{i \geq 1} D_{i}(G)=\{1\}$.

Proposition 1. ( $\overline{\mathrm{BB}}, \overline{\mathrm{FR}} 2])$ Let $A, C$ be two groups such that $C$ acts on $A$ by automorphisms. If $A \rtimes C$ is an almost-direct product then $\Gamma_{m}(A \rtimes C)=\Gamma_{m}(A) \rtimes \Gamma_{m}(C)$ and $D_{m}(A \rtimes C)=D_{m}(A) \rtimes D_{m}(C)$.

Corollary 2. The almost-direct product of two residually nilpotent (torsion free) groups is residually nilpotent (torsion free).

The pure braid group $P_{n}$ is an almost-direct product of free groups ([FR1]). Since free groups are residually torsion-free nilpotent $[\mathbb{F}]$, it follows from Corollary 2 that pure braid groups are residually torsionfree nilpotent (see also [FR2]).

\section{PResentations FOR PURE BRAid GROUPS ON SURFACES}

Let $\Sigma_{g}$ be an oriented closed surface of genus $g$. Let $\mathcal{X}=\left\{x_{1}, \ldots, x_{n}\right\}$ be a set of $n$ distinct points (punctures) in the interior of $\Sigma_{g}$. A pure geometric braid on $\Sigma_{g}$ based at $\mathcal{X}$ is a collection $\left(\psi_{1}, \ldots, \psi_{n}\right)$ of $n$ disjoint paths (called strands) on $\Sigma_{g} \times[0,1]$ which run monotonically with $t \in[0,1]$ and such that $\psi_{i}(0)=\left(x_{i}, 0\right)$ and $\psi_{i}(1)=\left(x_{i}, 1\right)$. Two pure braids are considered to be equivalent if they are isotopic relatively to the base points. The usual product of paths defines a group structure on the equivalence classes of braids. This group, which is isomorphic to $P_{n}\left(\Sigma_{g}\right)$, does not depend on the choice of $\mathcal{X}$.

We recall a group presentation for pure braid groups of oriented closed surfaces $\left[\mathrm{B}\right.$. In the following we set $[a, b]=a^{-1} b^{-1} a b, a^{b}=b^{-1} a b$ and ${ }^{b} a=b a b^{-1}$ and we use the convention that $W=\prod_{i=m}^{n} f(i)$ with $n<m$ implies that $W=1$.

Theorem 3. ( $\mathbb{B})$ Let $g \geq 1$ and $n \geq 2$. The group $P_{n}\left(\Sigma_{g}\right)$ admits the following presentation:

Generators: $\left\{A_{i, j} \mid 1 \leq i \leq 2 g+n-1,2 g+1 \leq j \leq 2 g+n, i<j\right\}$. 
Relations:

$\begin{array}{ll}\text { (PR1) } & A_{i, j}^{-1} A_{r, s} A_{i, j}=A_{r, s} \text { if }(i<j<r<s) \text { or }(r+1<i<j<s), \\ & \text { or }(i=r+1<j<s \text { for even } r<2 g \text { or } r>2 g) ; \\ \text { (PR2) } & A_{i, j}^{-1} A_{j, s} A_{i, j}=A_{i, s} A_{j, s} A_{i, s}^{-1} \text { if }(i<j<s) ; \\ \text { (PR3) } & A_{i, j}^{-1} A_{i, s} A_{i, j}=A_{i, s} A_{j, s} A_{i, s} A_{j, s}^{-1} A_{i, s}^{-1} \text { if }(i<j<s) ; \\ \text { (PR4) } & A_{i, j}^{-1} A_{r, s} A_{i, j}=A_{i, s} A_{j, s} A_{i, s}^{-1} A_{j, s}^{-1} A_{r, s} A_{j, s} A_{i, s} A_{j, s}^{-1} A_{i, s}^{-1} \\ & \text { if }(i+1<r<j<s) \text { or } \\ & (i+1=r<j<s \text { for odd } r<2 g \text { or } r>2 g) ; \\ \text { (ER1) } & A_{r+1, j}^{-1} A_{r, s} A_{r+1, j}=A_{r, s} A_{r+1, s} A_{j, s}^{-1} A_{r+1, s}^{-1} \\ & \text { if } j<s, r \text { odd and } r<2 g ; \\ \text { (ER2) } & A_{r-1, j}^{-1} A_{r, s} A_{r-1, j}=A_{r-1, s} A_{j, s} A_{r-1, s}^{-1} A_{r, s} A_{j, s} A_{r-1, s} A_{j, s}^{-1} A_{r-1, s}^{-1} \\ & \text { if } j<s, r \text { even and } r<2 g . \\ & (k=1, \ldots, n) . \\ & \left(\prod_{i=1}^{g}\left[A_{2 i-1,2 g+k}^{-1}, A_{2 i, 2 g+k}\right]\right)^{-1}=\prod_{l=2 g+1}^{2 g+k-1} A_{l, 2 g+k} \prod_{j=2 g+k+1}^{2 g+n} A_{2 g+k, j} \\ \text { (TR) } & (k=1, \ldots\end{array}$

As a representative of the generator $A_{i, j}$, we may take a geometric braid whose only non-trivial (non-vertical) strand is the $(j-2 g)$ th one. In Figure 1, we illustrate the projection of such braids on the surface $\Sigma_{g}$ (see also Figure 8 of $[\mathbb{B}]$ ). Some misprints in Relations (ER1) and (ER2) of Theorem 5.1 of [B] have been corrected. Remark also that in $[\mathbb{B}]$ was used the convention $[a, b]=a b a^{-1} b^{-1}$.

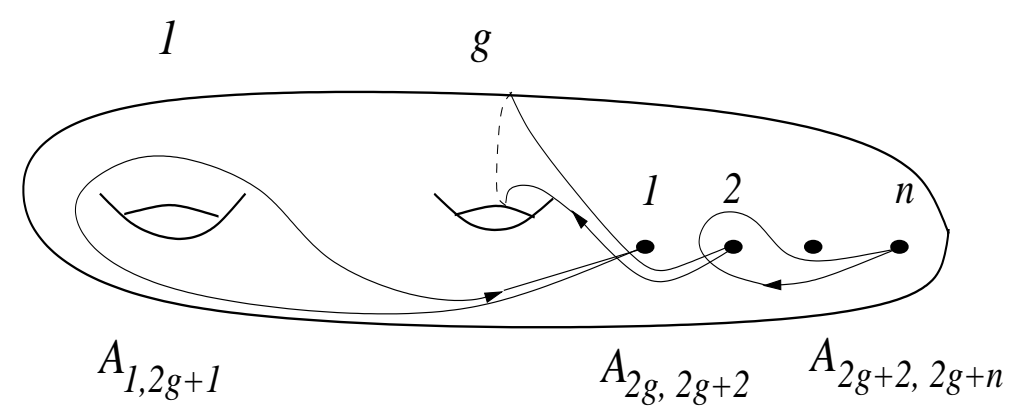

FigurE 1. Projection of representatives of the generators $A_{i, j}$. We represent $A_{i, j}$ by its only non-trivial strand. 
As remarked in the proof of Theorem 5.1 of $[\mathbb{B}]$, using above list of relations we can write any element of type $A_{i, j} A_{p, q} A_{i, j}^{-1}$ for $2 g+$ $1 \leq j<q \leq 2 g+n$ as a word on the generators $A_{1, q}, \ldots, A_{q-1, q}$. Therefore starting from the group presentation in Theorem 3, we can obtain the following group presentation for $P_{n}\left(\Sigma_{g}\right)$, where, in respect to Theorem 3, we set $A_{2 r-1,2 g+j}=a_{j, r}$ and $A_{2 r, 2 g+j}=b_{j, r}$ for $r=1, \ldots, g$ and $j=1, \ldots, n$ and $A_{2 g+i, 2 g+j}=\tau_{i, j}$ for $1 \leq i<j \leq n$.

Proposition 4. Let $g \geq 1$ and $n \geq 2$. The group $P_{n}\left(\Sigma_{g}\right)$ admits the following presentation:

Generators: $\left\{a_{j, k}, b_{j, k}, \tau_{p, q} \mid 1 \leq k \leq g, 1 \leq k \leq n, 1 \leq p<q \leq n\right\}$.

Relations:

$$
\begin{aligned}
& \text { (I-a) } \quad{ }^{a_{j, k}} c_{l, m}=c_{l, m} \quad \text { for } \quad c_{l, m}=a_{l, m}, b_{l, m} \text { if }(m<k) \text {; } \\
& \text { (I-b) } \quad{ }^{b_{j, k}} c_{l, m}=c_{l, m} \quad \text { for } \quad c_{l, m}=a_{l, m}, b_{l, m} \text { if }(m<k) \text {; } \\
& (I-\tau 1) \quad{ }^{\tau_{s, j}} c_{l, m}=c_{l, m} \quad \text { for } \quad c_{l, m}=a_{l, m}, b_{l, m} \text {; } \\
& \left(I-\tau \text { Q) } \quad \tau_{p, j} \tau_{s, l}=\tau_{s, l}\right. \\
& \text { (II-a) } \quad{ }^{a_{j, k}} a_{l, k}=a_{l, k}^{\tau_{j, l}} \text {; } \\
& (I I-b) \quad{ }^{b}, k b_{l, k}=b_{l, k}^{\tau_{j, l}} \\
& (I I-\tau) \quad \tau_{s, j} \tau_{s, l}=\tau_{s, l}^{\tau_{j, l}} \\
& \text { (III-a1) } \quad{ }^{a_{j, k}} c_{l, m}={ }^{\left[\tau_{j, l}, a_{l, k}\right]} c_{l, m} \quad \text { for } \quad c_{l, m}=a_{l, m}, b_{l, m} \text { if }(k<m) \text {; } \\
& (I I I-a 2){ }^{a_{j, k}} \tau_{s, l}={ }^{\left[\tau_{j, l}, a_{l, k}\right]} \tau_{s, l} \text {; } \\
& (I I I-b 1) \quad{ }^{b j, k} c_{l, m}={ }^{\left[\tau_{j, l}, b_{l, k}\right]} c_{l, m} \quad \text { for } \quad c_{l, m}=a_{l, m}, b_{l, m} \text { if }(k<m) \text {; } \\
& \text { (III-b2) }{ }^{b_{j, k}} \tau_{s, l}={ }^{\left[\tau_{j, l}, b_{l, k}\right]} \tau_{s, l} \text {; } \\
& (I I I-\tau) \quad{ }^{\tau_{s, j}} \tau_{p, l}={ }^{\left[\tau_{j, l}, \tau_{s, l}\right]} \tau_{p, l} \\
& (I V-a) \quad{ }^{a_{j, k}} \tau_{r, l}=\tau_{r, l} \text {; } \\
& (I V-b) \quad b_{j, k} \tau_{r, l}=\tau_{r, l} \text {; } \\
& (I V-\tau) \quad{ }^{\tau_{p, j}} \tau_{r, l}=\tau_{r, l} \text {; } \\
& (V-a) \quad{ }^{a_{j, k}} \tau_{j, l}=\left[\tau_{j, l}, a_{l, k}\right] \tau_{j, l} \text {; } \\
& (V-b) \quad b_{j, k} \tau_{j, l}=\left[\tau_{j, l}, b_{l, k}\right] \tau_{j, l} \\
& (V-\tau) \quad \tau_{s, j} \tau_{j, l}=\left[\tau_{j, l}, \tau_{s, l}\right] \tau_{j, l} \\
& \text { (ER1) } \quad{ }^{a_{j, k}} b_{l, k}=\tau_{j, l}^{-1} b_{l, k}\left[a_{l, k}, \tau_{j, l}\right] \text {; } \\
& \text { (ER2) } \quad{ }^{b_{j, k}} a_{l, k}=a_{l, k} \tau_{j, l} \text {; } \\
& \text { (TR) } \prod_{i=1}^{g}\left[a_{l, i}^{-1}, b_{l, i}\right]=\left(\prod_{w=1}^{l-1} \tau_{w, l} \prod_{d=l+1}^{n} \tau_{l, d}\right)^{-1} \text {; }
\end{aligned}
$$


where $1 \leq m, k \leq g$ and $1 \leq s<p<j<r<l<q \leq n$ (with $s, p, q, r$ and $l$ possibly absent).

Remark also that Proposition 14 can be proven directly. In fact drawing corresponding braids, one can verify that above relations hold in $P_{n}\left(\Sigma_{g}\right)$. To prove that they form a complete set of relations it is sufficient to repeat the arguments in the proof of Theorem 5.1 of $\mathbb{B}$.

\section{The STRUCTURE OF PURE BRAID GROUPS OF CLOSED SURFACES}

Let $p: P_{n}\left(\Sigma_{g}\right) \rightarrow \pi_{1}\left(\Sigma_{g}\right)$ be the map which forgets all strands except the first one. This map is induced from the forgetting map at the level of corresponding configuration spaces and ker $p$ is isomorphic to $P_{n-1}\left(\Sigma_{g, 1}\right)$, the pure braid group on $n-1$ strands of the oriented surface $\Sigma_{g, 1}$ of genus $g$ with one boundary component (see for instance [GG]).

In the following we provide an algebraic section $s$ for $p: P_{n}\left(\Sigma_{g}\right) \rightarrow$ $\pi_{1}\left(\Sigma_{g}\right)$, where $P_{n}\left(\Sigma_{g}\right)$ has the presentation given in Proposition 1 and we show that $s$ induces a structure of almost-direct product on $P_{n}\left(\Sigma_{g}\right)$.

In Theorem 1 of GG it was shown that $p$ admits a geometric section (i.e. induced from a section on the geometric level of corresponding configuration spaces). In [GG] it was also given an algebraic definition of such section using another group presentation for $P_{n}\left(\Sigma_{g}\right)$ (provided in Corollary 8 of $G G$ ).

Before stating the main result of the paper we need a preliminary Lemma. In the following, we will set $T_{l, q}=\prod_{d=l+1}^{q} \tau_{l, d}$ for $1 \leq l<q \leq$ $n$.

Lemma 5. The following identities:

(1) $\quad\left(\prod_{d=l}^{q} c_{d, k}\right) T_{l, q}=T_{l, q}\left(\prod_{d=l}^{q} c_{d, k}\right)$ for $\quad c_{d, k}=a_{d, k}, b_{d, k}$

(2) ${ }^{b_{l, k}}\left(\prod_{d=l+1}^{q} a_{d, k}\right)=\left(\prod_{d=l+1}^{q} a_{d, k}\right) T_{l, q}$,

hold in $P_{n}\left(\Sigma_{g}\right)$ for $1 \leq l<q \leq n$ and $1 \leq k \leq g$.

Proof. We provide an algebraical verification of these identities that can be also verified drawing corresponding braids.

We prove the first identity, in the case of $c_{d, k}=a_{d, k}$ (the case of $c_{d, k}=b_{d, k}$ is analogous). First we remark that for $1 \leq i<l<j \leq n$ and $1 \leq k \leq g$,

$$
a_{l, k} \tau_{i, l} \tau_{i, j} \tau_{i, l}^{-1} a_{l, k}^{-1}=\underline{a_{l, k} \tau_{l, j}^{-1} \tau_{i, j} \tau_{l, j} a_{l, k}^{-1}}=\tau_{l, j}^{-1} \tau_{i, j} \tau_{l, j},
$$

respectively because of relation II- $\tau$, relation III-a2 and relation V-a. Therefore applying once more relation II- $\tau$ we obtain that 
ON RESIDUAL PROPERTIES OF PURE BRAID GROUPS OF CLOSED SURFACE\$

(A) $\quad\left(\tau_{i, l}^{-1} a_{l, k} \tau_{i, l}\right) \tau_{i, j}\left(\tau_{i, l}^{-1} a_{l, k}^{-1} \tau_{i, l}\right)=\underline{\tau_{i, l}^{-1} \tau_{l, j}^{-1} \tau_{i, j} \tau_{l, j} \tau_{i, l}}=\tau_{i, j}$

holds in $P_{n}\left(\Sigma_{g}\right)$ for $1 \leq i<l<j \leq n$ and $1 \leq k \leq g$. Now we claim that for $1 \leq l<q \leq n$ and $1 \leq k \leq g$,

$$
\text { (B) } \quad\left(\prod_{d=l}^{q} a_{d, k}\right) \tau_{l, q}=\tau_{l, q}\left(\prod_{d=l}^{q} a_{d, k}\right)
$$

In fact,

$\left(\prod_{d=l}^{q} a_{d, k}\right) \tau_{l, q}\left(\prod_{d=l}^{q} a_{d, k}\right)^{-1}=\left(\prod_{d=l+1}^{q-1} a_{l, k} a_{d, k}\right) \cdot{ }^{a_{l, k}}\left(a_{q, k} \tau_{l, q} a_{q, k}^{-1}\right) \cdot\left(\prod_{d=l+1}^{q-1} a_{l, k} a_{d, k}\right)^{-1}=$ and by relation II-a,

$$
=\left(\prod_{d=l+1}^{q-1} a_{d, k}^{\tau_{l, d}}\right) \cdot \underline{{ }^{a_{l, k}}\left(a_{q, k} \tau_{l, q} a_{q, k}^{-1}\right)} \cdot\left(\prod_{d=l+1}^{q-1} a_{d, k}^{\tau_{l, d}}\right)^{-1}=
$$

and therefore applying relations II-a and V-a,

$$
=\left(\prod_{d=l+1}^{q-1} a_{d, k}^{\tau_{l, d}}\right)\left(\tau_{l, q}\right)\left(\prod_{d=l+1}^{q-1} a_{d, k}^{\tau_{l, d}}\right)^{-1} .
$$

Applying identity (A) we obtain that $\left(\tau_{l, d}^{-1} a_{d, k} \tau_{l, d}\right) \tau_{l, q}\left(\tau_{l, d}^{-1} a_{d, k}^{-1} \tau_{l, d}\right)=$ $\tau_{l, q}$ for $l+1 \leq d<q \leq n$ and therefore

$$
\left(\prod_{d=l+1}^{q-1} a_{d, k}^{\tau_{l, d}}\right)\left(\tau_{l, q}\right)\left(\prod_{d=l+1}^{q-1} a_{d, k}^{\tau_{l, d}}\right)^{-1}=\tau_{l, q}
$$

and the identity (B) is proved. Thus, because of relation I- $\tau 1$ the following identity holds:

$$
\left(\prod_{d=l}^{q} a_{d, k}\right) T_{l, q}=a_{l, k} a_{l+1, k} \tau_{l, l+1} a_{l+2, k} \tau_{l, l+2} \cdots a_{q, k} \tau_{l, q}
$$

Hence applying relation (B) recursively we obtain that

$$
\left(\prod_{d=l}^{q} a_{d, k}\right) T_{l, q}=\prod_{d=l+1}^{q} \tau_{l, d} \prod_{d=l}^{q} a_{d, k}=T_{l, q}\left(\prod_{d=l}^{q} a_{d, k}\right) .
$$


The second identity is easier to verify. From relations ER2 and I- $\tau 1$ in Proposition 4 one obtains the following identities:

$$
\begin{aligned}
& b_{l, k} \quad\left(\prod_{d=l+1}^{q} a_{d, k}\right)=\prod_{d=l+1}^{q}\left(a_{d, k} \tau_{l, d}\right)=a_{l+1, q}\left(\prod_{d=l+1}^{q-1}\left(\tau_{l, d} a_{d+1, k}\right)\right) \tau_{l, q}= \\
& =\cdots=\prod_{d=l+1}^{q}\left(a_{d, k}\right) T_{l, q} .
\end{aligned}
$$

Let $\left\langle c_{1}, d_{1}, \ldots, c_{g}, d_{g} \mid \prod_{i=1}^{g}\left[c_{i}^{-1}, d_{i}\right]=1\right\rangle$ be a group presentation for $\pi_{1}\left(\Sigma_{g}\right)$; the morphism $p: P_{n}\left(\Sigma_{g}\right) \rightarrow \pi_{1}\left(\Sigma_{g}\right)$ can be defined algebraically as follows: $p\left(a_{1, k}\right)=c_{k}$ and $p\left(b_{1, k}\right)=d_{k}$ for $1 \leq k \leq g$ and $p\left(a_{j, k}\right)=$ $p\left(b_{j, k}\right)=\tau_{p, q}=1$ elsewhere.

Theorem 6. The exact sequence

$$
1 \rightarrow P_{n-1}\left(\Sigma_{g, 1}\right) \rightarrow P_{n}\left(\Sigma_{g}\right) \rightarrow \pi_{1}\left(\Sigma_{g}\right) \rightarrow 1
$$

splits and $P_{n}\left(\Sigma_{g}\right) \simeq P_{n-1}\left(\Sigma_{g, 1}\right) \rtimes \pi_{1}\left(\Sigma_{g}\right)$ is almost-direct product of $P_{n-1}\left(\Sigma_{g, 1}\right)$ and $\pi_{1}\left(\Sigma_{g}\right)$.

Proof. Let us define a set-section $s: \pi_{1}\left(\Sigma_{g}\right) \rightarrow P_{n}\left(\Sigma_{g}\right)$ as follows: $s\left(c_{k}\right)=T_{1, n} a_{1, k} T_{1, n}^{-1}$ and $s\left(d_{k}\right)=T_{1, n} b_{1, k} T_{1, n}^{-1}$ for $1 \leq k<g, s\left(c_{g}\right)=$ $\prod_{d=1}^{n} a_{d, g} T_{1, n}$ and $s\left(d_{g}\right)=b_{1, g}$. In order to prove that $s$ is a well-defined morphism it suffices to prove that

$$
\prod_{i=1}^{g}\left[s\left(c_{i}\right)^{-1}, s\left(d_{i}\right)\right]=s\left(\prod_{i=1}^{g}\left[c_{i}^{-1}, d_{i}\right]\right)=1 .
$$

From Lemma 5 and relation TR in Proposition 4 one deduces the following identities:

$$
\begin{aligned}
& \prod_{i=1}^{g}\left[s\left(c_{i}^{-1}\right), s\left(d_{i}\right)\right]=T_{1, n} \prod_{i=1}^{g-1}\left[a_{1, i}^{-1}, b_{1, i}\right] T_{1, n}^{-1} \prod_{d=1}^{n} a_{d, g} T_{1, n} b_{1, g}^{-1}\left(\prod_{d=1}^{n} a_{d, g} T_{1, n}\right)^{-1} b_{1, g}= \\
& \quad=T_{1, n} \prod_{i=1}^{g-1}\left[a_{1, i}^{-1}, b_{1, i}\right] \prod_{d=1}^{n} a_{d, g} b_{1, g}^{-1}\left(\prod_{d=1}^{n} a_{d, g} T_{1, n}\right)^{-1} b_{1, g}=T_{1, n} \prod_{i=1}^{g-1}\left[a_{1, i}, b_{1, i}^{-1}\right] \times \\
& \quad \times a_{1, g} b_{1, g}^{-1} \cdot{ }^{b_{1, g}}\left(\prod_{d=2}^{n} a_{d, g}\right) \cdot\left(\prod_{d=2}^{n} a_{d, g} T_{1, n}\right)^{-1} a_{1, g}^{-1} b_{1, g}=T_{1, n} \prod_{i=1}^{g}\left[a_{1, i}^{-1}, b_{1, i}\right]=1
\end{aligned}
$$

and therefore $s: \pi_{1}\left(\Sigma_{g}\right) \rightarrow P_{n}\left(\Sigma_{g}\right)$ is a well-defined morphism and (3) splits. 
Now, remark that from relation ER2 we deduce the following identities:

$$
\begin{gathered}
\tau_{w, l}=\left[a_{l, g}, b_{w, g}^{-1}\right] \quad \text { for } 1 \leq w<l \leq n ; \\
\tau_{l, d}=\left[a_{d, g}, b_{l, g}^{-1}\right] \quad \text { for } 1 \leq l<d \leq n .
\end{gathered}
$$

Therefore, from relations TR and ER2 in Proposition 4 we obtain that the following relation

$$
\begin{gathered}
\tau_{1, l}^{-1}=\prod_{w=2}^{l-1} \tau_{w, l} \prod_{d=l+1}^{n} \tau_{l, d} \prod_{i=1}^{g}\left[a_{l, i}^{-1}, b_{l, i}\right]= \\
=\prod_{w=2}^{l-1}\left[a_{l, g}, b_{w, g}^{-1}\right] \prod_{d=l+1}^{n}\left[a_{d, g}, b_{l, g}^{-1}\right] \prod_{i=1}^{g}\left[a_{l, i}^{-1}, b_{l, i}\right]
\end{gathered}
$$

holds in $P_{n}\left(\Sigma_{g}\right)$ for $2 \leq l \leq n$ and then $\tau_{1, l} \in \Gamma_{2}(\operatorname{ker} p)$ for $l=2, \ldots, n$.

Fixing $j=1$, relations in Proposition 14 provide the action by conjugacy of $a_{1, k}, b_{1, k}$ for $1 \leq k \leq g$ on the set $\mathcal{A}=\left\{a_{j, k}, b_{j, k}, \tau_{p, q} \mid 1 \leq k \leq g\right.$, $2 \leq j \leq n$ and $1 \leq p<q \leq n\}$ and using the fact that $\tau_{1, l} \in \Gamma_{2}(\operatorname{ker} p)$ for $l=2, \ldots, n$ one can easily check that

$$
{ }^{a_{1, k}} h \equiv h \quad \bmod \Gamma_{2}(\operatorname{ker} p)
$$

and

$$
b_{1, k} h \equiv h \quad \bmod \Gamma_{2}(\operatorname{ker} p)
$$

for $1 \leq k \leq g$ and $h \in \mathcal{A}$.

Hence the action of $\pi_{1}\left(\Sigma_{g}\right)$ on the abelianisation of ker $p$ is trivial. In fact, let $h \in \mathcal{A}$. It follows from previous congruences that

$$
s\left(c_{k}\right) h={ }^{T_{1, n} a_{1, k} T_{1, n}^{-1}} h \equiv h \quad \bmod \Gamma_{2}(\operatorname{ker} p)
$$

and

$$
{ }^{s\left(d_{k}\right)} h={ }^{T_{1, n} b_{1, k} T_{1, n}^{-1}} h \equiv h \quad \bmod \Gamma_{2}(\operatorname{ker} p),
$$

for $1 \leq k \leq g-1$ and $h \in \mathcal{A}$. On the other hand since also $\prod_{d=2}^{n} a_{d, g} \in \operatorname{ker} p$, one derives that

$$
{ }^{s\left(c_{g}\right)} h=\prod_{d=1}^{n} a_{d, g} T_{1, n} h \equiv{ }^{a_{1, g}} h \equiv h \quad \bmod \Gamma_{2}(\operatorname{ker} p) .
$$

Finally $s\left(d_{g}\right)=b_{1, g}$ and therefore

$$
{ }^{s\left(d_{g}\right)} h \equiv h \quad \bmod \Gamma_{2}(\operatorname{ker} p) .
$$

Since $\mathcal{A}$ is a complete set of generators for ker $p$, the action of $\pi_{1}\left(\Sigma_{g}\right)$ is trivial on the abelianisation of ker $p$. 
Corollary 7. The group $P_{n}\left(\Sigma_{g}\right)$ is residually torsion free nilpotent for $n \geq 1$ and $g>0$.

Proof. The group $P_{1}\left(\Sigma_{g}\right)$ is isomorphic to $\pi_{1}\left(\Sigma_{g}\right)$ which is residually free and therefore residually torsion free nilpotent. In the case $n>1$ the claim is a straightforward consequence of Corollary 2, Theorem 6 and the fact that $P_{n-1}\left(\Sigma_{g, 1}\right)$ is residually torsion free nilpotent for $n \geq 1$ and $g, p>0$ BGG.

We remark that was already proven in BGG that $P_{n}\left(\mathbb{T}^{2}\right)$ is residually torsion free nilpotent and that $P_{n}\left(\mathbb{S}^{2}\right)$ is residually nilpotent but not residually torsion free nilpotent. The group $P_{n}\left(\Sigma_{g}\right)$ was proven to be bi-orderable in [Go].

We recall that residual torsion free nilpotence implies the residual $p$-finiteness. We recall that the Torelli group $\mathcal{T}\left(\Sigma_{g}\right)$ of the surface $\Sigma_{g}$ is defined as the kernel of the natural action of the mapping class group of $\Sigma_{g}$ on $H_{1}\left(\Sigma_{g}\right)$. Let $\mathcal{P}$ a set of $n$ distinct point on $\Sigma_{g}$. According to $[\mathrm{Pa}]$, let $\mathcal{T}_{p}\left(\Sigma_{g}, \mathcal{P}\right)$ be the kernel of the action of the $n$-th punctured mapping class group of $\Sigma_{g}$ on $H_{1}\left(\Sigma, \mathbb{F}_{p}\right)$. The group $\mathcal{T}_{p}\left(\Sigma_{g}, \mathcal{P}\right)$ is residually $p$-finite $[\mathrm{Pa}]$. Since $P_{n}\left(\Sigma_{g}\right)$ can be easily realised as subgroups of $\mathcal{T}_{p}\left(\Sigma_{g}, \mathcal{P}\right)$, one derives another proof of the residually $p$-finiteness of $P_{n}\left(\Sigma_{g}\right)$.

Finally, let us remark that Theorem 6 could be proved using the group presentation of $P_{n}\left(\Sigma_{g}\right)$ and the algebraic section proposed in [GG], but related computations would become much more involved.

\section{BRAID GROUPS ON 2 STRANDS}

Let us recall a well known on the braid group $B_{n}$.

Proposition 8. Let $B_{n}$ be the Artin braid group on $n \geq 3$ strands. Then $\Gamma_{1}\left(B_{n}\right) / \Gamma_{2}\left(B_{n}\right) \cong \mathbb{Z}$ and $\Gamma_{2}\left(B_{n}\right)=\Gamma_{3}\left(B_{n}\right)$.

A similar result holds for Artin-Tits group of finite type BGG.

Now, let $\Sigma_{g}$ be a closed oriented surface of genus $g>0$ and let $B_{n}\left(\Sigma_{g}\right)$ be the braid group on $n$ strands of $\Sigma_{g}$. The main result has been to determine all lower central quotients of surface braid groups on at least 3 strands. In particular, it was proven that $\Gamma_{2}\left(B_{n}\left(\Sigma_{g}\right)\right) / \Gamma_{3}\left(B_{n}\left(\Sigma_{g}\right)\right) \simeq$ $\mathbb{Z}_{n-1+g}$ and that $\Gamma_{3}\left(B_{n}\left(\Sigma_{g}\right)\right)=\Gamma_{4}\left(B_{n}\left(\Sigma_{g}\right)\right)$ for $n \geq 3$.

Since for $n=1$ we have that $B_{1}(\Sigma)=\pi_{1}(\Sigma)$, which is residually free, in order to complete the study of lower central series of braid groups of closed surfaces we need to consider the case of 2 strands. In the case of the torus $\mathbb{T}^{2}$, in BGG was proved that $B_{2}\left(\mathbb{T}^{2}\right)$ is residually nilpotent using essentially the fact that this group is a central extension of $\mathbb{Z}_{2} * \mathbb{Z}_{2} * \mathbb{Z}_{2}$. 
Actually the residually nilpotence of $B_{2}\left(\Sigma_{g}\right)$, for any $g>0$, is a consequence of Theorem 6 and of the following result of Gruenberg. As above we set $\mathcal{F P}$ the class of groups having the group-theoretic property $\mathcal{P}$.

Lemma 9. ( $\mathrm{Gr})$ Let $\mathcal{F P}$ be one of following classes:

(i) the class of solvable groups;

(ii) the class of finite groups;

(iii) the class of p-finite groups for a given prime number $p$.

Let $P \in \mathcal{F} \mathcal{P}$ and suppose that $H$ is residually $\mathcal{P}$. Then for each extension $1 \rightarrow H \rightarrow G \rightarrow P \rightarrow 1$, the group $G$ is residually $\mathcal{P}$.

Corollary 10. The group $B_{2}\left(\Sigma_{g}\right)$ is residually 2-finite. In particular $B_{2}\left(\Sigma_{g}\right)$ is residually nilpotent.

Proof. Let $S_{n}$ the symmetric group on $n$ elements. We recall that $P_{n}\left(\Sigma_{g}\right)$ can be realized as the kernel of the canonical projection $\pi$ : $B_{n}\left(\Sigma_{g}\right) \rightarrow S_{n}$. Since $S_{2}=\mathbb{Z}_{2}$ and $P_{2}\left(\Sigma_{g}\right)$ is residually torsion free, the hypothesis of Lemma 9 are fulfilled and the claim follows.

We recall that pure braid groups of surfaces with non empty boundary are residually torsion free nilpotent: then, from Lemma 9 it follows that also braid groups on 2 strands of surfaces with non empty boundary are residually 2 -finite.

Therefore, for any oriented and connected surface $\Sigma$ of positive genus, $B_{2}(\Sigma)$ is residually nilpotent. In BGG was also proved that $B_{2}\left(\mathbb{T}^{2}\right)$ is not residually torsion free nilpotent neither bi-orderable. We don't know if $B_{2}(\Sigma)$ is bi-orderable when $\Sigma$ is an oriented and connected surface of positive genus different from $\mathbb{T}^{2}$.

\section{REFERENCES}

[BB] P. Bellingeri and V. Bardakov, Combinatorial properties of virtual braids, math.GT/0609563.

[B] P. Bellingeri, On presentations of surface braid groups, J. Algebra, 274 (2004), 543-563.

[BGG] P. Bellingeri, J. Guaschi and S. Gervais, Lower central series for surface braids, math.GT/0512155.

[CCP] D. Cohen, F. Cohen and S. Prassidis, Centralizers of Lie algebras associated to the descending lower central series of certain poly-free groups, math.GT/0603470.

[FR1] M. Falk and R. Randell, The lower central series of a fiber type arrangement Invent. Math., 82 (1985), 77-88.

[FR2] M. Falk and R. Randell, Pure braid groups and products of free groups. Contemp. Math., 78(1988), 217-228. 
[F] R. H. Fox Free differential calculus I: Derivation in the Free Group Ring, Ann. of Math., 57(1953), 547-560.

[GG] D. Gonçalves and J. Guaschi, The roots of the full twist for surface braid groups, Math. Proc. Camb. Phil. Soc. 137 (2004), 307-320.

[Go] J. González-Meneses, Ordering pure braid groups on closed surfaces, Pacific J. Math. 203 (2002), no 2, 369-378.

[GP] J. González-Meneses et L. Paris, Vassiliev Invariants for braids on surfaces, Trans. A.M.S. $356 \mathrm{n}^{\circ} 1$ (2004), 219-243.

[Gr] K. W. Gruenberg, Residual properties of infinite soluble groups, Proc. London Math. Soc. 7 (1957), 29-62.

[MKS] W. Magnus, A. Karrass, D. Solitar, Combinatorial group theory, Interscience Publishers, New York, 1996.

[MR] R. Mura and A. Rhemtulla, Orderable groups, Lecture Notes in Pure and Applied Mathematics 27, Marcel Dekker, New York, 1977.

[P] S. Papadima, The universal finite-type invariant for braids, with integer coefficients, Topology Appl., 118 (2002), 169-185.

[Pa] L. Paris, Residually $p$ properties of mapping class groups and surface groups, math.GT/0703703.

[Pas] I. Bir S. Passi, Group rings and Their Augmentation Ideals, Lecture Notes In Mathematics 715, Springer Veralg, Berlin Heidelberg New York, 1979.

Sobolev Institute of Mathematics, 630090 Novosibirsk, Russia

E-mail address: bardakov@math.nsc.ru

Univ. Milano Bicocca, Dipartimento Matematica e Applicazioni, 20126 Milan, Italy

E-mail address: paolo.bellingeri@unimib.it 